\title{
Short-term outcome after total hip arthroplasty using dual-mobility cup: report from Lithuanian Arthroplasty Register
}

\author{
Sarunas Tarasevicius $^{1} \cdot$ Alfredas Smailys $^{1} \cdot$ Kazimieras Grigaitis $^{1} \cdot$ Otto Robertsson $^{2}$ • \\ Justinas Stucinskas ${ }^{1}$
}

Received: 13 December 2016 / Accepted: 26 December 2016 / Published online: 12 January 2017

(C) The Author(s) 2017. This article is published with open access at Springerlink.com

\begin{abstract}
Purpose The purpose of the study was to investigate how the use of dual-mobility cups (DMCs) affected the risk of revision due to dislocation as well as overall risk of revision compared with a conventional total hip arthroplasty (THA) system in the short term.

Methods A total of 12,657 primary THAs were registered from the start of 2011 to the end of 2014. 620 THAs were with DMCs. For comparison, we included all registered THAs with Exeter cup and a cemented Exeter stem combined with 28-mm femoral head. Patients were followed up with respect to revision and/or death until 1 January 2016. For survival analysis, we used revision as an endpoint. Cox proportional hazards models were used to analyse the influence of various covariates (age, gender, surgical approach, THA model and pre-operative diagnosis).

Results Of the 620 dual-mobility THAs and 2170 Exeter THAs, 100 had been revised. The overall unadjusted cumulative revision rate (CRR) for any reason of revision at five years after surgery was $3.9 \%$ in the dual-mobility group and $5.2 \%$ in the Exeter group. Cox regression analysis, adjusting for age, gender, THA type, surgical approach and pre-operative diagnosis, showed that the risk of revision was less in patients operated with DMCs and in patients having their operation for osteoarthritis.
\end{abstract}

Sarunas Tarasevicius

sarunas.tarasevicius@gmail.com

1 Department of Orthopedics, Lithuanian University of Health Sciences, Kaunas, Lithuania

2 Department of Orthopedics, Lund University and Lund University Hospital, Lund, Sweden
Conclusion The DM implant had a lower short-term complication rate than a conventional well defined THA. Low dislocation rate suggests that it is a good choice for high risk patients.

Keywords Dual mobility cup $\cdot$ Hip $\cdot$ Arthroplasty $\cdot$ Register

\section{Introduction}

Dislocations after total hip arthroplasty surgery (THA) are a serious problem and according to the Lithuanian Arthroplasty Register they are the most common reason for re-operations during the first two years after primary surgery [1]. The risk factors for prosthetic dislocation after THA are multifactorial and may be patient-specific (gender, age, abductor deficiency, mental illness etc.) or related to the surgery (approach, malposition, femoral head diameter) [2-5]. Using larger femoral heads and dual mobility systems has become popular in an effort to reduce the dislocation rate. The dual articulation cup concept was developed in 1974 combining the "low friction" principle of THA popularized by Charnley [6] with the McKee-Farrar concept of a larger diameter femoral head in order to enhance implant stability [7]. The goal was to achieve the greatest possible range of motion in a stable environment as well as to reduce wear. Dual mobility cups have been found to be an good option in revision THA especially when performed for recurrent dislocation [8]. Concerning use of DMCs for primary THA in general, a number of studies have shown that the concept significantly reduces the dislocation rate [9]. However, the overall survival varies among studies ranging from $81.4 \%$ to $96.3 \%$ at 15 years and with a dislocation rate between $0 \%$ and $1 \%$ [10]. We are only aware of one report from a National Arthroplasty Register regarding 287 primary DM hips of 78,098 THAs implanted in Sweden [5]. The 
authors reported that the use of DMCs resulted in a clinically relevant reduction in the dislocation rate.

In Lithuania, use of DMCs in primary THA has been relatively popular. We investigated how their use affected the risk of revision due to dislocation as well as their overall risk of revision as compared with a conventional, well-established THA system.

\section{Materials and methods}

The Lithuanian Arthroplasty Register (LAR) was established in 2011 and registers all primary and revision procedure for hips and knees. Data is collected via the Internet and is based on a minimal data set, as suggested by the European Arthroplasty Register [11], which includes patient age, sex, pre-operative diagnosis, surgical approach and part numbers of components used. Completeness of the register was validated by comparing data with that in the Implant Usage Database of the State Patient Fund and was found to be $86 \%$ for primaries and $99 \%$ for revisions. As with many other registers, the LAR defines revision as a second operation after an arthroplasty in which implant components are exchanged, removed or added. A total of 12,657 primary THAs were registered from the start of 2011 to the end of 2014. Of these, 620 used DMCs (both cemented and cementless), which were included in the study. The Avantage cup (Biomet) was used in 542 cases and the Quattro (Lepine group) in 78. In combination with the cups, a number of different stem types were used on the femoral side.

As comparison, we included all THAs using the polyethylene Exeter cup and a cemented Exeter stem with a 28-mm femoral head, but this implant combination was the one most frequently used during the study period. Patients were followed up with respect to revision and/or death until 1 January
2016. The Lithuanian National Census Register was used to obtain information on patients who died before the end of the follow-up period. The study was approved by the national ethical committee (No. BE-2-17).

\section{Statistical analysis}

For descriptive statistics, we used means, ranges and $95 \%$ confidence intervals (CI). Follow-up started on the day of primary THA and ended on the day of revision, death or 1 December 2015, whichever came first. Cumulative revision rate (CRR) curves were produced using the life table method using monthly intervals. CIs were calculated using the Wilson quadratic equation with Greenwood and Peto effective sample-size estimates [12]. Cox regression was used to compare the risk of revision of the DM group with that of the Exeter group, adjusting for differences in age, sex, surgical approach and whether the patient had been operated for OA. A $p$ value $<0.05$ was considered significant. In the case of bilaterally operations, both hips were included, but it has been reported that this has little effect on survival in register studies [14].

\section{Results}

Median follow-up time was $2.5(0-5)$ years. Demographic data is presented in Table 1. Osteoarthritis was the most common primary diagnosis for THA.

Of 620 DM THAs, 573 were implanted using a posterior approach and the remaining 47 using an anterolateral approach. Of the Exeter hips, 2074 were implanted using a posterior approach and 96 using an anterolateral approach. At the end of follow-up, 100 revisions had been performed. The distribution of revisions is presented in Table 2. Prosthetic
Table 1 Patient distribution according to diagnoses and demographic data

\begin{tabular}{llll}
\hline Variables & $\begin{array}{l}\text { Cemented DM, } \\
n=321\end{array}$ & $\begin{array}{l}\text { Cementless DM, } \\
n=299\end{array}$ & $\begin{array}{l}\text { Exeter cemented system, } \\
n=2170\end{array}$ \\
\hline $\begin{array}{l}\text { Age (years) } \\
\text { Gender }\end{array}$ & $68(14)$ & $58(12)$ & $68(11)$ \\
$\begin{array}{l}\text { Diagnosis } \\
\text { Osteoarthritis }\end{array}$ & F 207, M 114 & F 176, M 123 & F 1383, M 787 \\
$\begin{array}{l}\text { Femoral neck fracture } \\
\text { Rheumatoid arthritis }\end{array}$ & 121 & 250 & 1614 \\
$\begin{array}{l}\text { Hip dysplasia } \\
\text { Post-traumatic }\end{array}$ & 3 & 12 & 365 \\
$\quad$ osteoarthritis & 9 & 1 & 6 \\
Avascular osteonecrosis & 16 & 7 & 38 \\
Other & 21 & 6 & 50 \\
\hline
\end{tabular}

$D M$ dual-mobility 
Table 2 Distribution of revisions for Exeter and dual-mobility total hip arthroplasty (DM THA)

\begin{tabular}{lllllll}
\hline Reasons for revisions & Infection & $\begin{array}{l}\text { Cup } \\
\text { loosening }\end{array}$ & $\begin{array}{l}\text { Stem } \\
\text { loosening }\end{array}$ & $\begin{array}{l}\text { Loosening of both } \\
\text { components }\end{array}$ & Dislocation & Other \\
\hline $\begin{array}{l}\text { Exeter cemented } \\
\text { system, } n=2170\end{array}$ & 6 & 18 & 1 & 0 & 52 & 9 \\
$\begin{array}{c}\text { Cemented DM, } \\
n=321\end{array}$ & 5 & 1 & 0 & 1 & 1 & 2 \\
$\begin{array}{c}\text { Cementless DM, } \\
n=299\end{array}$ & 1 & 0 & 0 & 0 & 3 & 0 \\
\hline
\end{tabular}

dislocation was the most common reason for revision. In the Exeter group, 52 of 86 revisions were for dislocation (60\%), while four of 14 were for dislocation in the DM group (29\%), of which three were intraprosthetic. Of the 2170 surgeries in the Exeter group, 556 (26\%) were for other diseases than OA, accounting for 23 of the 52 revisions for dislocation (44\%). In the DM group, 250 of 620 (40\%) were for diseases other than $\mathrm{OA}$, accounting for one of the four revisions for dislocation.

The overall unadjusted CRR for any reason at five years after surgery was $5.2 \%$ (95\% CI 3.9-17.7) for the Exeter group and 3.9\% (CI 2.1-51.7), for DM group (Fig. 1). Cox regression analysis, adjusting for age, gender, THA type, surgical approach and pre-operative diagnosis showed that the risk of revision was less in patients operated upon with DMCs and in patient having their operation for osteoarthritis. The effects of surgical approach, gender and age were not statistically significant (Table 3). A separate analysis for patients having been operated upon for OA only showed a CRR for any reason of $4.5 \%$ (CI 3.1-18.8) for the Exeter and 2.5\% (CI 1.0-54.1) for the DM group (Fig 2), while Cox regression analysis showed no significant effects (THA type, age, gender, surgical approach).

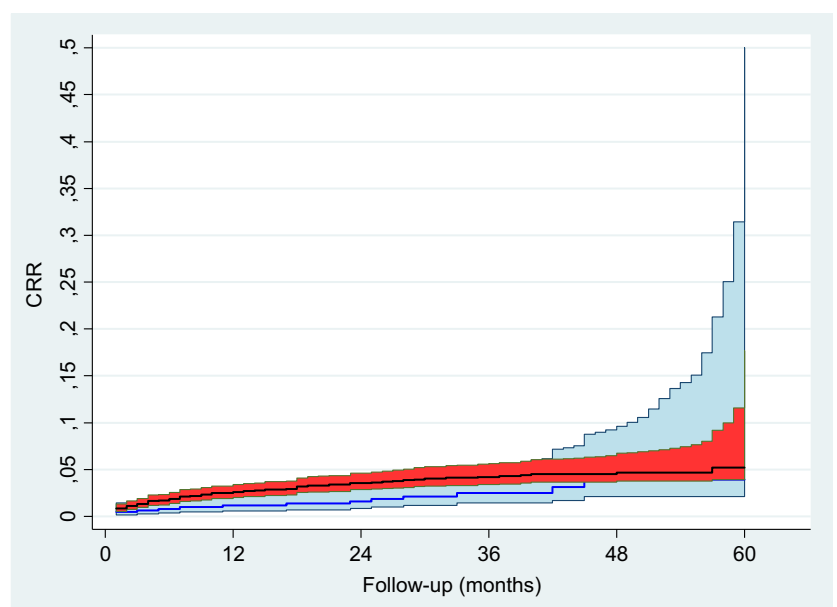

Fig. 1 Unadjusted cumulative revision rate (CRR) with 95\% confidence interval (CI) for all patients using any reason of revision as endpoint. Exeter CRR is shown as a black line with a red CI and the dualmobility (DM) group CRR with a blue line and light blue CI

\section{Discussion}

To our knowledge, this is the largest series of DMCs derived from a national arthroplasty register. Of all DMC patients, $44 \%$ had other diagnosis than OA, compared with $26 \%$ of patients in the Exeter group, which might indicate that Lithuanian surgeons have tended to use DMCs for more complex cases, which may have negatively affected outcomes for this group. However, at five years, the CRR for all reasons of revision was $4 \%$ for the 620 primary hips using DMCs compared with $5.4 \%$ for the Exeter group.

DMCs are reported to have a high rate of loosening $[10,13]$ and a higher rate of osteolysis and cup loosening in younger patients and cases of childhood disease sequelae [13]. Worries about these issues have limited the use of DMCs. Our shortterm comparison of DMCs to that of a well-documented THA system (Exeter) shows that the DM THA performed at least as well in the short term. We also found that surgery for diagnoses other than OA had an increased risk of revision, suggesting that dual articular cups could be considered for non-OA patients, e.g. for THA due to femoral neck fractures.

DMCs have mainly been used worldwide in patients considered prone to dislocation or in revisions for dislocations $[8$, 9]. Thus, it is likely that our nationwide database of patients receiving DMCs included patients with a higher-than-average

Table 3 Cox regression data. Men used as reference for women; Exeter total hip arthroplasty (THA) used as reference for dual-mobility (DM) THA; posterior approach is used as reference for anterolateral; osteoarthritis used as a reference for all other preoperative diagnoses combined

\begin{tabular}{llll}
\hline Variables & RR & $95 \%$ CI & $P$ value \\
\hline Age & 1.00 & $0.99-1.02$ & 0.504 \\
Gender & 0.67 & $0.44-1.01$ & 0.059 \\
THA model & 0.54 & $0.30-0.96$ & 0.035 \\
Surgical approach & 1.27 & $0.56-2.92$ & 0.570 \\
Pre-operative diagnosis & 1.99 & $1.33-2.99$ & 0.001 \\
\hline
\end{tabular}

$R R$ revision rate, $C I$ confidence interval 


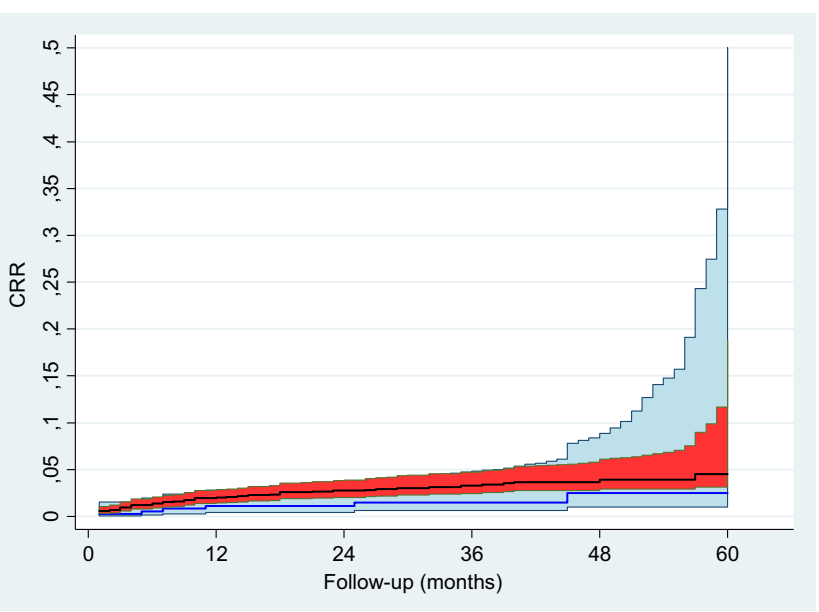

Fig. 2 Unadjusted cumulative revision rate (CRR) with 95\% confidence intervals $(\mathrm{CI})$ for only patients operated for osteoarthritis (OA) using any reason of revision as endpoint. Exeter CRR shown as black line with a red CI; dual-mobility (DM) group CRR shown with a blue line and light blue $C I$

risk of postoperative dislocation. Still, among the 620 primary DM hips, four revisions only were due to dislocation (of which three were intraprosthetic), compared with 52 revisions for dislocation in the Exeter group of 2170 hips.

Also to be considered is that after a primary arthroplasty, a revision for dislocation in conventional primary THA is usually not done before a patient has dislocated two to three times; thus, the RR for dislocation is lower than the true dislocation rate. However, dislocations of DMCs are often intraprosthetic, requiring surgery after the first event. Thus, the RR for dislocation is probably not much lower than the true dislocation rate.

A study from a single hospital in Lithuania found low RRs for dislocation with the DM cup in high-risk femoral neck fracture patients [9]. Similarly, Combes at al. investigated 2480 primary THA with DMCs and reported $0.88 \%$ dislocation rate, which is far below the rates reported with conventional THA [14]. Similar results were observed in our current register study, where revision rates due to dislocation in DMCs were $0.7 \%$ compared with $2.4 \%$ in the Exeter group.

A limitation of our study was selection bias due to the tendency to select DM systems for high-risk patients, especially those with femoral neck fractures (Table 1). This should theoretically have a negative effect on outcomes in DMCs; however, our findings were contradictory, finding that DMCs had a lower RR compared with the Exeter THA. Another limitation of our study is the relatively short followup; however, even these results are promising.

We conclude that DM implant had a low short-term complication rate, comparable with that of a conventional, welldefined THA. The especially low dislocation rate makes it a reasonable choice for high-risk patients, while longer-term results are needed before it can be recommended for general use.
Open Access This article is distributed under the terms of the Creative Commons Attribution 4.0 International License (http:// creativecommons.org/licenses/by/4.0/), which permits unrestricted use, distribution, and reproduction in any medium, provided you give appropriate credit to the original author(s) and the source, provide a link to the Creative Commons license, and indicate if changes were made.

\section{References}

1. Tarasevicius S, Cebatorius A, Valaviciene R, Stucinskas J, Leonas L, Robertsson O (2014) First outcome results after total knee and hip replacement from the Lithuanian arthroplasty register. Medicina (Kaunas, Lithuania) 50:87-91. doi:10.1016/j.medici.2014.06.004

2. Berry DJ, von Knoch M, Schleck CD, Harmsen WS (2005) Effect of femoral head diameter and operative approach on risk of dislocation after primary total hip arthroplasty. J Bone Joint Surg Am 87: 2456-2463. doi:10.2106/jbjs.d.02860

3. Enocson A, Hedbeck CJ, Tidermark J, Pettersson H, Ponzer S, Lapidus LJ (2009) Dislocation of total hip replacement in patients with fractures of the femoral neck. Acta Orthop 80:184-189. doi: $10.3109 / 17453670902930024$

4. Werner BC, Brown TE (2012) Instability after total hip arthroplasty. World J Orthopedics 3:122-130. doi:10.5312/wjo.v3.i8.122

5. Hailer NP, Weiss RJ, Stark A, Karrholm J (2012) The risk of revision due to dislocation after total hip arthroplasty depends on surgical approach, femoral head size, sex, and primary diagnosis. An analysis of 78,098 operations in the Swedish Hip Arthroplasty Register. Acta Orthop 83:442-448. doi:10.3109/17453674.2012.733919

6. Charnley J (1972) The long-term results of low-friction arthroplasty of the hip performed as a primary intervention. J Bone Joint Surg British Vol 54:61-76

7. McKee GK, Watson-Farrar J (1966) Replacement of arthritic hips by the McKee-Farrar prosthesis. J Bone Joint Surg British Vol 48: 245-259

8. Cross MB (2015) Dual mobility acetabular components for revision THA. J Orthop Traumatol: Off J Italian Soc Orthop Traumatol 16: 21-22. doi:10.1007/s10195-015-0347-x

9. Tarasevicius S, Busevicius M, Robertsson O, Wingstrand H (2010) Dual mobility cup reduces dislocation rate after arthroplasty for femoral neck fracture. BMC Musculoskelet Disord 11:175. doi:10.1186/1471-2474-11-175

10. Philippot R, Farizon F, Camilleri JP, Boyer B, Derhi G, Bonnan J, Fessy MH, Lecuire F (2008) Survival of cementless dual mobility socket with a mean 17 years follow-up. Rev Chir Orthop Reparatrice Appar Mot 94:e23-27. doi:10.1016/j.rco.2007.10.013

11. Sadoghi P, Leithner A, Labek G (2013) Overcoming boundaries of worldwide joint arthroplasty registers: the European Arthroplasty Register minimal dataset. J Arthroplast 28: 1327-1328. doi:10.1016/j.arth.2013.02.023

12. Dorey F, Nasser S, Amstutz H (1993) The need for confidence intervals in the presentation of orthopaedic data. J Bone Joint Surg 75:1844-1852

13. Lautridou C, Lebel B, Burdin G, Vielpeau C (2008) Survival of the cementless Bousquet dual mobility cup: Minimum 15-year followup of 437 total hip arthroplasties. Rev Chir Orthop Reparatrice Appar Mot 94:731-739. doi:10.1016/j.rco.2008.06.001

14. Combes A, Migaud H, Girard J, Duhamel A, Fessy MH (2013) Low rate of dislocation of dual-mobility cups in primary total hip arthroplasty. Clin Orthop Relat Res 471:3891-3900. doi:10.1007/s11999-013-2929-3 